\title{
Length-independent voltage fluctuations in small conductors
}

\author{
A M JAYANNAVAR \\ Department of Physics, Indian Institute of Science, Bangalore 560012, India \\ MS received 15 February 1988
}

Abstract. The mean-squared voltage fuctuation of a disordered conductor of length $L$ smaller than the phase coherence length $L_{\phi}$, is independent of the distance between the probes. We obtain this result using the voltage additivity and the known results for the conductance fluctuation. Our results complement the recent theoretical and experimental findings.

Keywords. Universal conductance fluctuations; phase coherence length.

PACS Nos $72 \cdot 10 ; 72 \cdot 15 ; 72 \cdot 90$

It has been shown both experimentally and theoretically that one can observe surprising, novel phenomena at low temperatures (Imry 1986a; Washburn and Webb 1986 ) in the quantum-transport regime, when the inelastic mean-free path $L_{\phi}$ (or the phase-breaking length) of an electron is comparable to the size of the sample. It is now well established that these phenomena are consequences of coherent interference effects because of elastic scattering by impurities (static disorder). Elastic scattering causes phase randomization; however, electrons do not lose their phase memory. On the contrary, inelastic scattering leads to a loss of phase memory. In disordered systems the length over which an electron can retain its phase memory $L_{\phi}$ can be much larger than the elastic mean-free path $L_{e}$. In certain disordered metals, at low temperatures ( $\simeq 1 \mathrm{~K}$ ), $L_{\phi}$ can be of the order of $\mu \mathrm{m}$ (or more depending on the resistance of the sample). Micron size devices have been fabricated using modern lithographic and electronbeam techniques. Some of the phenomena observed in these systems include (Washburn and Webb 1986) the normal state Aharonov-Bohm effect in disordered metallic rings, universal conductance fluctuations in metallic systems and the nonadditivity and non-self-averaging nature of quantum ohmic resistance in thin disordered wires (Kumar and Jayannavar 1985).

Recently it has been shown experimentally (Benoit et al 1987) that the voltage fluctuations, of an ensemble of disordered conductors, measured between the two voltage probes (in a four-probe geometry) are independent of distance $x$ between the probes, when $x$ is less than $L_{\phi}$. The root-mean-squared (rms) value of these fluctuations is given by $\Delta V_{\phi}=I R_{\phi}^{2}\left(e^{2} / h\right)$. Here $I$ is the current flowing through the conductor and $R_{\phi}$ the classical average resistance of the conductor of length $L_{\phi}$. When the voltage fluctuations are measured over a distance larger than $L_{\phi}$, then one can treat the system as if it is composed of independent subsystems of length $L_{\phi}$; i.e., voltage fluctuations in different subsystems are uncorrelated. Hence the square of the voltage fluctuations is additive and we get $\Delta V=\Delta V_{\phi}\left(L / L_{\phi}\right)^{1 / 2}$, where $\Delta V_{\phi}$ is the rms voltage fluctuation across a sample of length $L_{\phi}$. This is in conformity with the experimental results (Washburn 
and Webb 1986). In all the experiments performed so far, one measures on a single sample. This corresponds to studying a single realization of a microscopic random potential out of an ensemble of macroscopically identical samples. Theoretical treatments based on the Landauer formula (Landauer 1970; Buttiker et al 1985) clearly indicate that, in the quantum transport regime $\left(L \leqslant L_{\phi}\right)$, the numerical value of the conductance is a sensitive functional of the underlying potential. Moreover in a metallic conduction regime (i.e., when the conductance $G \geqslant\left(e^{2} / h\right)$ ) the conductance of a sample will fluctuate if one varies the chemical potential or the magnetic field in the sample. The rms value of these conductance fluctuations is universal, independent of both the degree of disorder and the sample size (Stone 1985; Lee and Stone 1985; Lee et al 1987; Altshuler 1985; Altshuler and Khmelnitskii 1985; Imry 1986b). The magnitude of $\Delta G=e^{2} / h$. In this regime it has been shown that changes in the chemical potential or the magnetic field (larger than some scales) cause fluctuations in conductance, which are statistically the same as the conductance of a sample with another impurity configuration. Hence experimentally, one effectively studies an ensemble of disordered conductors by varying either the chemical potential or the magnetic field in one sample.

To understand the length-independent voltage fluctuations as mentioned above, Buttiker (1987) has given a complete scattering formalism in which voltage probes are treated as a part of a conductor. Benoit et al (1987) have explained their results by invoking voltage additivity and Onsager symmetries (Buttiker 1986) in a four-probe set-up. We give here a simple complementary treatment based on general arguments similar in spirit to those on Benoit et al (1987). In our treatment we invoke voltage additivity and the known results for conductance and its fluctuations in metallic and in insulating regime.

Consider first a three-probe set-up as shown in figure 1. A constant current $I$ is applied to a long conductor of length $L$ connected to infinite reservoirs, with chemical potentials $\mu_{1}$ and $\mu_{2}\left(\mu_{1}-\mu_{2}=\mathrm{eV}\right)$ at the end points 1 and 2 respectively. The length of this conductor is less than the phase coherence length, i.e., no inelastic scattering occurs inside the conductor. The phase incoherence which leads to irreversibility occurs only inside the reservoirs. In such a situation the phase coherence length is the distance between the reservoirs $\left(L=L_{\phi}\right)$. In figure 1 we have located a voltage probe at a distance $L_{1}$ from one end (1) of the wire. Now the measured voltages across distances $L_{1}, L_{2}$ and $L$ are $V_{13}\left(L_{1}\right), V_{32}\left(L_{2}\right)$ and $V_{21}\left(L=L_{1}+L_{2}\right)$ respectively. Voltage additivity requires

$$
V_{13}\left(L_{1}\right)+V_{32}\left(L_{2}\right)+V_{21}(L)=0 .
$$
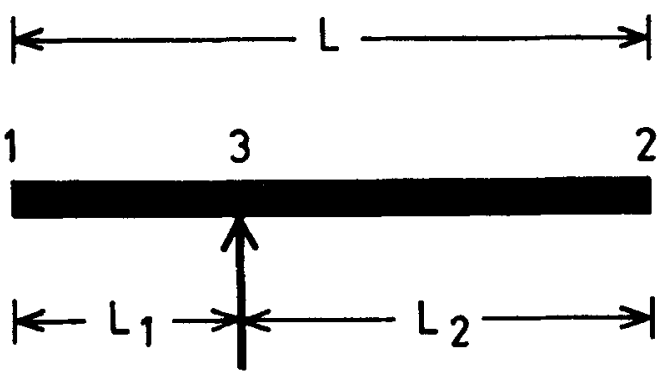

Figure 1. Configuration of three-probe voltage measurement. 
Using the general property (triangular inequality) that the rms fluctuation $(\Delta V)$ of one term is always less than or equal to the sum of two other terms (Benoit et al 1987) we have

$$
\begin{aligned}
& \Delta V_{21}\left(L=L_{1}+L_{2}\right) \leqslant \Delta V_{13}\left(L_{1}\right)+\Delta V_{32}\left(L_{2}\right) \\
& \Delta V_{13}\left(L_{1}\right) \leqslant \Delta V_{21}(L)+\Delta V_{32}\left(L_{2}\right) \\
& \Delta V_{32}\left(L_{2}\right) \leqslant \Delta V_{21}(L)+\Delta V_{13}\left(L_{1}\right)
\end{aligned}
$$

In analogy with the classical addition of voltage fluctuations, the measured $\Delta V$ between two probes should depend only on the distance $x$ between the probes; thus we set $\Delta V(x)=x^{\alpha}$. It can be shown rigorously that $0 \leqslant x \leqslant 1$. The upper limit is obtained by setting $L_{1}=L_{2}$ in equation (2a); the lower by taking $L_{1} \rightarrow 0$ in (2b). This shows that length dependence of voltage fluctuations must be bounded by a constant value or a linear variation.

Let the conductor we are considering be in a metallic regime (i.e., conductance $\left.G \geqslant e^{2} / h\right)$. In this regime the average resistance $R$ of the sample scales linearly with the length, i.e. $R=\rho_{0} L$. The rms value of voltage fluctuations across the conductor is given by $\Delta V(L)=I R^{2} \Delta G$, where $\Delta G$ is the rms value of the conductance fluctuation. Now we invoke the result of the theory of universal conductance fluctuations (Stone 1985; Lee and Stone 1985; Lee et al 1987; Altshuler 1985; Altshuler and Khmelnitskii 1985; Imry 1986b) which states that $\Delta G$ is universal across the sample (in a two-probe geometry) and is given by $e^{2} / h$. Taking this value of $\Delta G$ across the sample of length $L$ (across the end points) we get $\Delta V_{21}^{\prime}(L)=I \rho_{0}^{2} L^{2}\left(e^{2} / h\right)$. Note that $\Delta V_{12}(L) \sim L^{2}$.

We will now show that the length independence of the voltage fluctuations (i.e. $\Delta V_{13}\left(L_{1}\right)$ and $\Delta V_{32}\left(L_{2}\right)$ is independent of $L_{1}$ and $L_{2}$ respectively) follows naturally by requiring $V_{21}(L) \sim L^{2}$ to be consistent with $0 \leqslant \alpha \leqslant 1$. This is seen best by letting $L_{1} \rightarrow$ $L-0^{+}$(i.e. letting $L_{1}$ tend arbitrarily close to $L$ ). Then we must have $\Delta V_{13}\left(L-0^{+}\right)=$ $\Delta V_{21}(L)$ which is equal to $I \rho_{2}^{0} L^{2}\left(e^{2} / h\right)$. Since, however, $\Delta V_{13}\left(L-0^{+}\right) \propto\left(L-0^{+}\right)^{\alpha}=$ $L^{\alpha}$ (as $L-0^{+}$is essentially $L$ ), we will require $\alpha=2$ (i.e. $\left.\Delta V_{13}\left(L_{1}\right) \sim L_{1}^{2}\right)$ which violates the inequality $0 \leqslant \alpha \leqslant 1$. The only way to recover consistency is to let $\alpha \rightarrow 0$ and set $\Delta V_{13}\left(L_{1}\right)=\Delta V_{32}\left(L_{2}\right)=\Delta V_{21}(L)$ itself. This clearly demonstrates that the potential fluctuation $\left(\Delta V_{13}\right.$ and $\left.\Delta V_{32}\right)$ depends only on the total length $L$ of the sample and not on the position of the voltage probes. This result can be trivially generalized for a fourprobe geometry. Our result implies that even while probing scales smaller than the phase coherence length, we will observe full quantum interference effects $u p$ to the phase coherence length. This sort of non-locality arises due to the wave nature of electrons which explore the distances upto phase coherence length before arriving at any two probes. This is clearly evident in recent experiments on non-local voltage fluctuations (Washburn and Webb 1986).

The results we have derived in the metallic regime also hold in the insulating regime: In the insulating regime the average resistance grows exponentially with the size of the sample (Kumar and Jayannavar 1986; Shapiro 1986) and we expect large anomalous conductance fluctuations. Using this fact in conjunction with the analysis given above it is easy to see that the voltage fluctuations are independent of distance $x$ between the probes, as long as $x$ is less than $L_{\phi}$. However, in a experimental situation one may not simulate an ensemble average by changing the chemical potential or the magnetic field in one sample. This is because in the insulating regime the level spacings are larger than 
level widths. Therefore for a sample drawn at random from the ensemble, the probability of the chemical-potential (Fermi level) coinciding with a level of the sample is arbitrarily small. Thus the sample specific fluctuations will be a non-resonant process (i.e. an electron ejected from the perfect lead at the Fermi level may not resonate with any energy level of the sample within the width of the level). On the other hand for a given sample as the chemical potential is tuned one must necessarily sweep through energy levels of the sample. The fluctuations will dominantly arise from such resonances (i.e. chemical potential lying within the half-width of the level). Hence strictly speaking the two need not be the same.

The author thanks Prof, N Kumar for many useful discussions.

\section{References}

Altshuler B L 1985 JETP Lett. 41648

Altshuler B L and Khmelnitskii 1985 JETP Lett. 42359

Benoit A, Umbach C P, Laibowitz R B and Webb R A 1987 Phys. Rev. Lett. 582343

Buttiker M, Imry Y, Landauer R and Pinhas S 1985 Phys. Rev. B31 6207

Buttiker M 1986 Phys. Rev. Lett. 571761

Buttiker M 1987 Phys. Rev. B35 4123

Imry Y 1986a in Directions in condensed matter physics (eds) G Grinstein and E Mazenko (Singapore: World Scientific Pub. Co.)

Imry Y 1986b Europhys. Lett. 1249

Kumar N and Jayannavar A M 1985 Phys. Rev. B32 3345

Kumar N and Jayannavar A M 1986 J. Phys. C19 L85

Landauer R 1970 Philos. Mag. 21863

Lee P A and Stone A D 1985 Phys. Rev. Lett. 551622

Lee P A, Stone A D and Fukuyama H 1987 Phys. Rev. B35 1039

Shapiro B 1986 Phys. Rev. B34 4394

Stone A D 1985 Phys. Rev. Lett. 542692

Washburn S and Webb R A 1986 Adv. Phys. 35375 (and references therein) 